\title{
Message framing and COVID-19 vaccination intention: Moderating roles of partisan media use and pre-attitudes about vaccination
}

\author{
Porismita Borah ${ }^{1,2}$ (D)
}

Accepted: 30 January 2022

(c) The Author(s), under exclusive licence to Springer Science+Business Media, LLC, part of Springer Nature 2022

\begin{abstract}
One of the ways to overcome the sheer devastation of the COVID-19 pandemic is to get vaccinated. However, vaccine hesitancy could be a significant barrier. The main purposes of the current study are to examine the impact of four types of theory-driven messages on COVID-19 vaccination intention and to understand the moderating role of partisan media use and vaccination attitudes. The study used a between-subject randomized online experiment with four conditions. The manipulation messages were presented as screenshots from the CDC's Facebook page. The total number of participants were 387 (female 43\%, mean age 37 years). The participants were from the U.S. and older than 18 years. The findings show that loss vs. gain message frames did not have any impact on COVID-19 vaccine intention. The moderating effects of conservative media and attitudes show that in general, those who consumed lower conservative media and held positive attitudes were higher on vaccine intention, and individual vs. collective frames did not have a strong impact. However, among those participants who scored high on conservative media use, and held negative vaccination attitudes, the individual frame had a higher impact on vaccine intention. The current study experimentally tested the intertwined relationships among message frames, partisan media use, and attitudes on vaccine intention. These relationships are critical considering the political nature of the pandemic.
\end{abstract}

Keywords COVID-19 $\cdot$ Message framing $\cdot$ COVID-19 vaccine intention $\cdot$ Partisan media use $\cdot$ Attitudes $\cdot$ Experiment . Vaccine hesitancy

\section{Introduction}

One of the ways to overcome the sheer devastation of the COVID-19 pandemic is to get vaccinated. However, vaccine hesitancy is a significant barrier for a successful implementation of the vaccine (Elkind, 2020). There is a strong anti-vaccination movement that dates back to the $1800 \mathrm{~s}$ (Blume, 2006). Most recently, even before the COVID-19 vaccination was available, the anti-vaccination movement had come out strongly against the COVID-19 vaccine (Jamison et al., 2020; Law, 2020). COVID-19 has also become a case of strong political polarization in the U.S. (Dong et al., 2020).

Porismita Borah

porismita@gmail.com

1 Edward R. Murrow College of Communication, Washington State University, Pullman, WA 99163, USA

2 Democracy Research Unit, Political Science, College of Law \& Public Administration, University of Salamanca, Salamanca, Spain
For example, in general, compared with liberals, conservatives often expressed skepticism towards the pandemic; Television hosts from FOX news mentioned that the public healthcare crisis is a "fraud" (Rupar, 2020); while former President Trump said the pandemic is a hoax (Franck, 2020). Motta et al., (2020) found that reliance on partisan sources led people to ignore the effective recommendations from medical experts.

The impact of news media use on vaccination behavior has been studied before (e.g., Gollust et al., 2016). For example, researchers have shown the impact of news media coverage on vaccine intention in the case of HPV vaccination (Leader et al., 2009; McRee et al., 2010). Besides, research has also shown that message frames (for example presenting the same information in terms of loss or gain) play an outsized role in vaccination attitudes and behavior (e.g., Nan, 2012a, b; Park, 2012). Although past research has examined the role of different frames on vaccination attitudes, few studies have examined the moderating role of partisan media on vaccine intention. Moreover, these relationships have not yet to be studied in terms of the COVID-19 pandemic. Considering 
the political nature of the pandemic, these relationships are critical. The current study fills this gap by examining the impact of message frames on COVID-19 vaccination intention. Particularly, the main purposes of the study are to examine the impact of four different types of message frames on COVID-19 vaccination intention and to understand the moderating role of two critical variables: partisan media use and vaccination attitudes. By doing so, this study experimentally tested the intertwined relationships among message frames, partisan media use, and attitudes on vaccine intention.

\section{Vaccine Promotion}

Vaccine refers to a "product that stimulates a person's immune system to produce immunity to a specific disease, protecting the person from that disease," while vaccination is "the act of introducing a vaccine into the body to produce immunity to a specific disease" (CDC, 2018). There is enough evidence to show that vaccination can reduce the risk of many infectious diseases (Thompson et al., 2018). Scientific evidence is solid regarding the benefits of vaccination including social benefits (UNICEF, 2019). Yet, vaccination hesitancy and misleading information around vaccines are increasing (WHO, n.d.). Over the years, vaccine promotion has summoned a colossal amount of scholarly attention and much of the research focuses on factors that leads to vaccination intention and behavior (Dillard, 2011; Ratanasiripong et al., 2013; Wheldon et al., 2018).

Scholars have examined how to decrease vaccine hesitancy and promote vaccines such that people take wellinformed decisions. Some studies have been devoted to understanding how information about vaccination may change people's attitudes towards vaccination. For example, Chanel et al. (2011) found that people's attitudes towards vaccination are stemmed from rational understanding. In general, scientific information had the capacity to induce positive influence on people's intention to vaccinate. Much of the research on vaccine intention and behavior have studied the impact of messages; employing message framing as a theoretical approach to understand the types of messages that could positively impact vaccine behavior.

\section{Framing}

Framing theory has been examined by a wide range of academic fields, such as sociology, psychology, cognitive science, politics, and communication (e.g., Borah, 2011; D'Angelo, 2002; Scheufele, 1999). Increasingly used in the realm of health communication (e.g., Nan, 2012a, b; Park, 2012) message framing is regarded as a helpful theoretical framework for health communication scholars, especially in the realm of health prevention science (e.g., Von Sikorski \& Matthes, 2019). Specifically, much attention has been paid to loss and gain frames (e.g., O'Keefe \& Jensen, 2008; O'Keefe \& Wu, 2012). Loss and gain frames have been drawn from the experimental work of Tversky and Kahneman's (1981) prospect theory. The same information presented in terms of losses or costs and gains or benefits can impact individuals' attitudes and behavior differently (Kahneman \& Tversky, 1984). On one hand, loss frames emphasize the unpleasant consequences or disadvantages if one does not comply with the recommended behaviors. On the other hand, gain frames underscore the desirable and pleasant consequences if one would comply with the recommended behaviors.

\section{Loss vs. Gain Frames and Vaccination}

Specifically, loss and gain frames have been widely used to understand vaccination behaviors. For example, Nan et al., (2012) examined loss vs. gain frames to explicate the underlying mechanism of H1N1 promotion message endorsement. Besides, loss vs. gain frames have also been employed to examine HPV vaccination uptake behaviors among young adults. In general loss frames have been shown to be slightly more persuasive for vaccination behavior (Nan, 2012a, b; Park, 2012). For example, with health pamphlets as manipulations, Nan (2012a) found that loss frames had a stronger persuasive effect. In addition, Nan (2012b) also found that compared with gain-framed messages, loss-framed messages were more effective in persuasion for participants who were avoidance-oriented in terms of predicting behavioral vaccination intentions. For participants who were approach-oriented, both gain frames and loss frames were equally persuasive (Nan, 2012b). In a recent study, Chen et al., (2021) showed that gain vs. loss frames did not matter for the COVID-19 vaccine intention. However, this study was conducted with participants from China where the "Chinese adults' attitudes toward COVID-19 vaccination were highly favorable, and the vaccination intention was high" (Chen et al., 2021 , p. 1). There are also few studies that did not show any effects of loss vs. gain framing on vaccination behavior (Gainforth et al., 2012), some showed equal impact of the two types of frames (Gerend \& Shepherd, 2012) in the U.S. context, but in general, past research shows a slight advantage of the loss frame. Based on this research, the first hypothesis is proposed:

H1. Participants in the loss frame condition will show higher intention to vaccinate compared to those in the gain frame condition. 


\section{Individual vs. Collective Frames}

Beyond psychological factors, societal factors such as culture, may often impact peoples' health decision-making processes. One important factor is individualism vs. collectivism, which explain the primary difference in the relationship between individuals and societies (Oyserman \& Lee, 2008; Triandis, 2001). Individualism refers to this cultural perspective that "the core unit is the individual" where societies help individuals to thrive (Oyserman \& Lee, 2008, p. 311). While in collectivism the "core unit is the group" and individuals fit themselves into existing societies (Oyserman \& Lee, 2008, p. 311). Research has demonstrated that these differences can impact health decision-making and individuals' health behavior. These factors may also impact how people react to health crises (Dutta, 2007).

In the case of vaccination, people with a collective worldview often consider collective health behavior (e.g., Finkelstein, 2010; Oyserman et al., 2002; Uskul, et al., 2009). While those people who have an individualistic worldview could focus on individual aspects (Böhm, et al., 2016; Earley, 1989). For example, Briley et al., (2017) conducted multiple studies to examine how individual's cultural views might impact their attitudes. Individualism and collectivism moderated people's health behavior including vaccination. These concepts from the literature on individualism and collectivism is discussed here to understand how they have been used in message design and framing of the health messages in our study.

Recent studies have used similar factors to examine the impact of individual vs. collective frames on peoples' decision-making (Pittman, 2020; Rabb et al., 2021). Pittman (2020) examined individuals' behavior regarding meat consumption, flu vaccination, and eco-friendly sunscreen. The findings revealed that the individual frame was more persuasive for flu vaccination behavior. Rabb and et al., (2021) provides evidence that individual appeals had a positive influence on COVID-19 vaccine intention. U.S. scores high on the individualism (Workman \& Lee, 2011) scale, and as a result in general people may focus on the individual interests (Workman \& Lee, 2011). With the help of literature, the second hypothesis is proposed:

H2. Participants in the individual frame condition will show higher intention to vaccinate compared to those in the collective frame condition.

\section{Partisan Media Use as a Moderator}

The use of news media could affect the uptake rate of vaccination (Gollust et al., 2016; Leader, et al., 2009). For example, by setting the agenda, the news media affect the focus and perspectives of both the public and policymakers (Yanovitzky, 2002). Indeed, prior studies have demonstrated that use of news media can impact people's attitudes towards vaccination. For example, in the case of HPV vaccination, previous studies showed that the news coverage of the goal of vaccination influenced the intentions of taking the vaccine (Leader et al., 2009; McRee et al., 2010). Besides, findings also revealed that there would be stronger support from the public if the news discussed the effectiveness of the vaccines as positive (Bigman et al., 2010). Moreover, some studies also found that people's attitudes towards the vaccination could be negatively influenced if the news focused on the controversial issues of the vaccination (Gollust et al., 2010). In another study conducted by Gollust et al., (2013), the results showed that news exposure was associated with stronger awareness for vaccination.

There have been drastic changes in the media landscape with the decline of traditional news media of the "big three" broadcast networks (Baum, 2011). In general, the mainstream media coverage of issues was considered similar (West, 2001). But the current media landscape is extremely partisan in the U.S. (Frisby, 2018). These partisan outlets offer opinions along with the news and these media organizations are biased in favor of a specific political opinion (Levendusky, 2013). This partisan slant of information is seen not only in matters of politics but health issues such as vaccination. For example, during the H1N1 pandemic conservative media outlets such as FOX news and conservative talk shows strongly criticized the H1N1 vaccine (Baum, 2011). Partisan media use can be particularly impactful for COVID-19 because this pandemic has been exceptionally political in nature (Dong et al., 2020; Rupar, 2020). In this scenario, examining the moderating role of partisan media on vaccine intention is essential.

H3. Partisan media consumption will moderate the relationship between message frames and COVID-19 vaccine intention such that, participants who consume more conservative media will show lower intention to vaccinate.

\section{Vaccination Attitudes as a Moderator}

Prior research has demonstrated the importance of attitudes toward vaccination in vaccination behavior (e.g., Guidry et al., 2020; Nyhan et al., 2014). A recent meta-analysis shows that attitude toward vaccination was the strongest predictor of vaccination behavior (Xiao \& Wong, 2020). In case of measles-mumps-rubella immunization, Nyhan et al. (2014) found that messages that advocate for vaccination are not always able to induce change in desirability towards the vaccine. Indeed, the researchers found that the impact of the message depended on pre-existing attitudes regarding 
the vaccination. Moreover, Lehmann et al. (2015) found that positive attitudes towards vaccination had significant impact on intention to vaccinate against influenza. Most recently in case of COVID-19 vaccine intention, Guidry et al., (2020) found that among other variables positive attitudes toward vaccination was an important predictor of vaccine intention. Based on this research, attitudes were considered an important moderator for intention to vaccinate. The final hypothesis is proposed:

H4. Attitudes toward vaccination will moderate the relationship between message frames and COVID-19 vaccine intention such that, participants' positive attitudes toward vaccination will show higher intention to vaccinate.

\section{Methods}

\section{Participants}

To test the hypotheses an online randomized experiment was conducted. The participants completed the survey via the Qualtrics software. The participants were randomly assigned to the four experimental groups (gain and individual frame; gain and collective frame; loss and individual frame; loss and collective frame) by Qualtrics. The participants for the study were recruited from Amazon's MTurk in July 2020. The total number of participants were 387 (female 43\%, mean age 37 years). A power analyses using GPower (Erdfelder et al., 1996) was conducted. $F$ was set at $0.25, \alpha$ err prob at 0.05 , and power $(1-\beta)$ at 0.95 . The minimum sample size needed was 210 . A sample of 387 was adequate to run the analyses. The participants were from the U.S. older than 18 years. The age range of the participants was from 21-73 years. Researchers use MTurk commonly for participant recruitment. MTurk participants are considered more diverse than student samples (Berinsky, 2012; Buhrmester, 2016). The data were collected after the study was approved as exempt by the Institutional Research Board of a large University in the U.S.

\section{Design and Procedure}

The study used a between-subject randomized experiment with four conditions. The topic of the study is the COVID-19 vaccination. The manipulation messages were presented as screenshots from the CDC's Facebook page. The participants were told that they will read a screenshot from the CDC's Facebook account in the next page. When they clicked the next page, they were exposed to one of the four messages. These messages were created in the Photoshop software. The posts were designed to look exactly like a Facebook message. Except the content of the message, the remaining information such as number of likes, shares, and image were kept consistent across the four messages. The post consisted of the CDC explaining about the COVID-19 vaccination when available. The content of the Facebook post is adapted from prior research (e.g., Nan, 2012a, 2012b). The manipulations were also designed with the help of older posts from the CDC on other vaccines such as flu or HPV. The study consisted of four conditions: gain and individual frame; gain and collective frame; loss and individual frame; loss and collective frame. The gain and individual frame consisted of 95 participants, the gain and collective frame consisted of 94 participants, loss and individual frame consisted of 100 participants, and loss and collective frame consisted of 98 participants. The gain frame described the benefits of getting the COVID-19 vaccine when available while the loss frame highlighted the costs of not getting the vaccine. The individual frame described the importance of taking care of one's health, while the collective frame highlighted the importance of thinking about the community's health. The stimulus materials are included in Appendix A.

\section{Measurements}

\section{Vaccine Intention}

The intention to vaccinate when the COVID-19 vaccine is available was captured with the help of three items and adapted from prior research (e.g., Nan, 2012a) on a 7-point Likert scale $(0=$ not at all to $6=$ extremely). Participants were asked to agree or disagree to the statements "How likely would you be to get the COVID-19 vaccine, as soon as it is available", "If you were faced with the decision of whether to get the COVID-19 vaccine today, how likely is it that you would choose to get the vaccine" and "How likely would you be to get the COVID-19 vaccine in the future when available." The three items were used to create an index $(\alpha=0.86, M=4.25, S D=1.32)$.

\section{Liberal Media Use}

This variable was also measured with three items asking participants about how often they use types of media sources on a five-point scale $(0=$ never to $4=$ multiple times a day). The items included are "The MSNBC cable news channel, website or app from the MSNBC cable news organization", "NPR radio broadcast, website or app from the NPR news organization" and "Liberal sources (such as Democracy Now, The Intercept, Mother Jones, The Nation, or Vox)." These items were used to create an index $(\alpha=0.76, M=2.14$, $S D=1.03)$. 


\section{Conservative Media Use}

Similarly, conservative media use was measured with three items asking participants about their media sources on a five-point scale $(0=$ never to $4=$ multiple times a day $)$. The items included are "The FOX News cable news channel, website or app from the FOX News cable news organization", "Conservative talk radio website or app from conservative talk radio (such as The Rush Limbaugh Show)" and "Conservative sources (such as The American Spectator, Breitbart, the Blaze, the Daily Caller, or the Daily Mail)." These three items were used to create an index $(\alpha=0.79, M=2.10, S D=1.08)$.

\section{Attitude Toward Vaccination}

A single item (e.g., Nan, 2012a) was used to measure attitudes toward the vaccination before the exposure to the manipulations. Participants were asked to respond on a 7 -point Likert scale $(0=$ strongly disagree to $6=$ strongly agree) to the item "vaccination has adverse side effects" $(M=3.5, S D=1.6)$. Those who scored higher showed higher negative attitude toward vaccination.

\section{Controls}

Although this was a randomized experimental design two variables were added as controls in the models. Considering the political nature of the COVID-19 pandemic, Party ID (51.2\% republican) was added a control. Adapted from prior research (e.g., Nan, 2012a) the second variable added was individuals' past flu shot behavior (41.3\% yes).

\section{Results}

\section{Manipulation Checks}

Once the participants had completed reading the Facebook post, they first answer the manipulation check question. The participants were asked to respond to the question "Which of the following comes closest to the position of the original Facebook post about the COVID-19 vaccine". The options were "The post highlighted the benefits of getting the COVID-19 vaccine for me when available", "The post highlighted the benefits of getting the COVID19 vaccine for the community when available", "The post highlighted the costs of not getting the COVID-19 vaccine for me when available" and "The post highlighted the costs of not getting the COVID-19 vaccine for the community when available." A chi-square analysis showed that the manipulation was effective $\left(\chi^{2}(3,387)=39.94, p<0.001\right)$.

\section{Findings}

Hayes' process model 1 (Hayes, 2013) was used to test the hypotheses. Findings from the first model shows that there was no significant difference between loss vs. gain frames on intention to vaccinate. Both main effects and interaction effects were not significant. Thus hypothesis 1 was not supported. Next, process model 1 was conducted to test $\mathrm{H} 2$ and $\mathrm{H} 3$. The overall model for conservative media use was marginally significant $\mathrm{F}(5,377)=2.07$, $p<0.07, R^{2}=0.03$, while the main effects of conservative media use $b=0.40, t(377)=2.05, p<0.01$ and the interaction between conservative media use and message frames $b=-0.28, t(377)=-2.27, p<0.01$ were both significant. The conditional effects of message frames on COVID-19 vaccination intention is stronger for participants' conservative media use one standard deviation above the mean $[b=-0.50, t(377)=-2.71, p<0.001]$ compared to those at the mean $[b=-0.20, t(377)=-1.55, p<0.12]$ and below the mean $[b=0.09, t(377)=0.51, p<0.60]$. Specifically, the interaction shows that for individuals who are higher users of conservative media, were willing to get vaccinated for the COVID-19 vaccine when they were exposed to the individual frame condition. While for individuals who are at the mean and below the mean this moderating effect was not significant (Fig. 1). The process model run with liberal media was not significant. Thus, $\mathrm{H} 2$ was supported, H3 was partially supported (Table 1 ).

Next, another process model was conducted with attitudes as a moderator, to test $\mathrm{H} 4$. The overall model for attitudes was significant $\mathrm{F}(5,377)=4.96, p<0.001$, $R^{2}=0.06$, as well as the interaction effect between attitudes about vaccination and message frames $b=-0.19, t$ $(377)=-2.39, p<0.01$. The conditional effects of message frames on COVID-19 vaccination intention is stronger for participants' who had negative attitudes about vaccination one standard deviation above the mean $[b=-0.54, t$ $(377)=-2.98, p<0.001]$ compared to those at the mean $[b=-0.23, t(377)=-1.82, p<0.07]$ and below the mean $[b=0.07, t(377)=0.40, p=0.68]$. Specifically, the interaction shows that for individuals who held higher negative attitudes, were willing to get vaccinated for the COVID-19 vaccine when they were exposed to individual frame message. While for individuals who are at the mean were the moderating effect was marginally significant and below the mean this moderating effect was not significant (Fig. 2). H4 was supported (Table 2). 
Fig. 1 Interaction effects between conservative media use

19 vaccination intention and message frames on COVID-

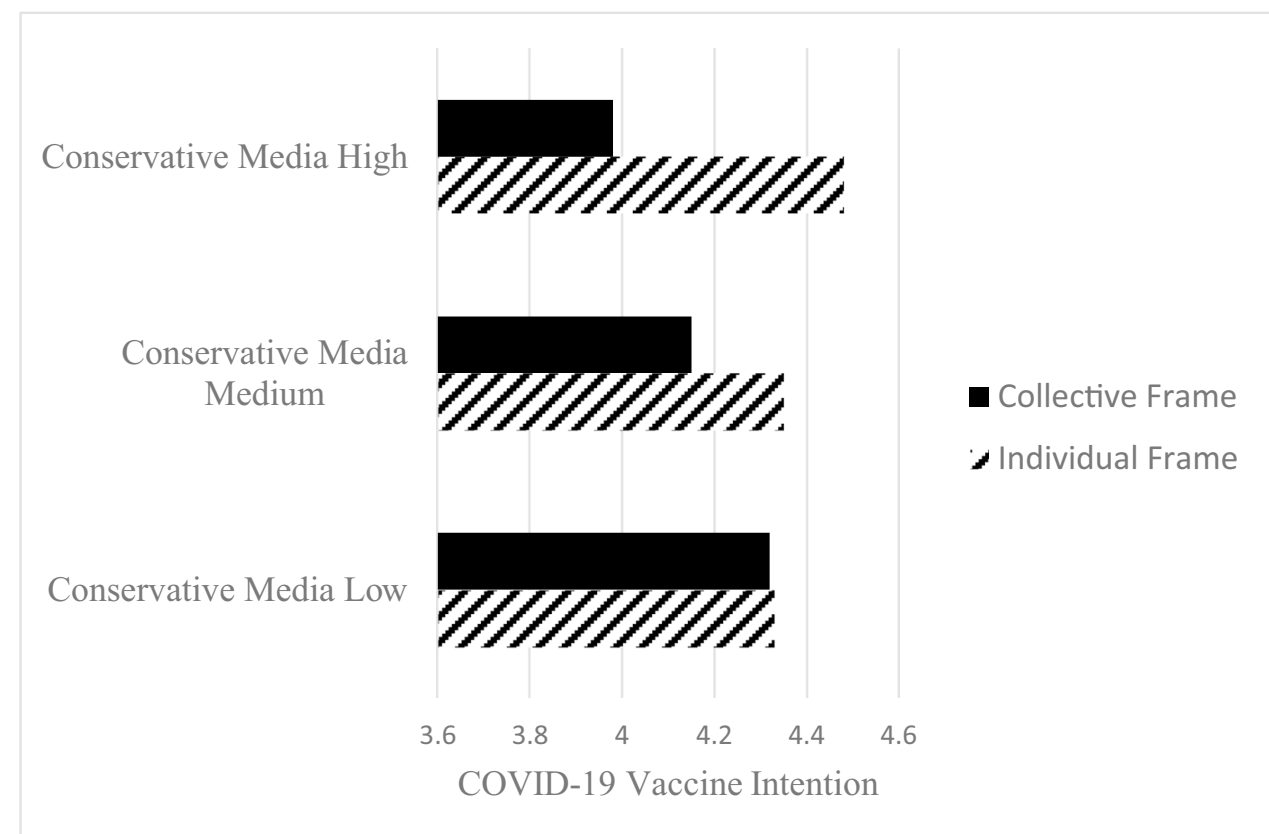

Table 1 Conditional effects of conservative media use and message frames on COVID-19 vaccination intention

\begin{tabular}{llll}
\hline Conservative Media & $t$ & LLCI & ULCI \\
\hline$-1 S D$ & .51 & -.2706 & .4608 \\
$M$ & -1.55 & -.4627 & .0541 \\
$+1 S D$ & -2.71 & -.8688 & -.1387 \\
\hline
\end{tabular}

$* p<.05 ; * * p<.01 ; * * * p<.001$

Fig. 2 Interaction effects between attitudes about vaccines and message frames on COVID-19 vaccination intention

\section{Discussion}

Even before the COVID-19 vaccine was available, the anti-vaccination movement had already come out strongly against the vaccine (Law, 2020). Moreover, the COVID-19 pandemic has become a deeply politicized issue in the U.S. (Dong et al., 2020). In this scenario understanding what messages might be helpful to increase vaccine intention is critical for public health and the dissemination of the vaccination.

The findings from the current study show that loss vs. gain frames did not have any impact on COVID-19 vaccine
Pre-attitudes High

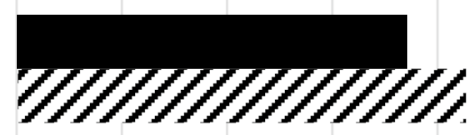

Pre-attitudes Medium

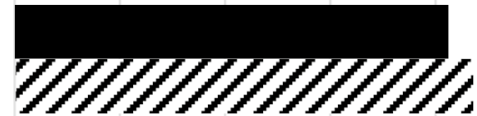

- Collective Frame

2 Individual Frame

Pre-attitudes Low

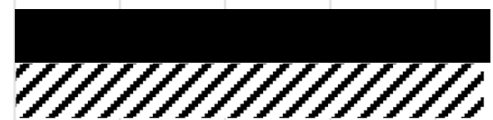

.

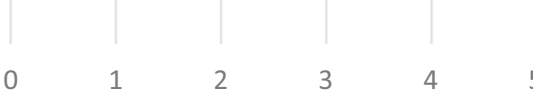

COVID-19 Vaccine Intention 
Table 2 Conditional effects of attitudes about vaccines and message frames on COVID-19 vaccination intention

\begin{tabular}{llll}
\hline Attitudes & $t$ & LLCI & ULCI \\
\hline$-1 S D$ & .40 & -.2857 & .4336 \\
$M$ & -1.82 & -.4893 & .0187 \\
$+1 S D$ & $-2.98 * * *$ & -.9036 & -.1854 \\
\hline
\end{tabular}

$* p<.05 ; * * p<.01 ; * * * p<.001$

intention. Prior research has been somewhat inconclusive about how loss vs. gain frames influence vaccination behavior. Although studies have shown that loss frames have a slightly stronger effect on vaccine behavior (e.g., Nan, 2012a, b; Park, 2012), there is research that shows no effect of these frames (e.g., Gainforth et al., 2012), or revealed equal influence of the two types of frames on vaccination behavior (Gerend \& Shepherd, 2012). A recent study from China showed that loss vs. gain frames did not matter for the COVID-19 vaccine (Chen et al., 2021). It is possible that the impact of loss vs. gain frames are specific to certain vaccines. It is also possible that these results might be different during a specific time of the COVID-19 timeline. This global pandemic has seen multiple waves of rising cases and number of deaths since March 2020. These factors could impact the findings.

The findings from the individual vs. collective frames and the moderating effects of partisan media use and attitudes reveal the complex nature of vaccination behavior. Prior research has indicated the role of partisan media on health behavior such as vaccination. For example, in the last pandemic before COVID-19, conservative media outlets such as FOX news had criticized the vaccine for H1N1(Baum, 2011). Similarly, in the case of the present pandemic conservative outlets have been spreading fear and false information about the COVID-19 vaccine (Bauder, 2021; Slisco, 2020). The moderating effects of conservative media in the present study shows that in general, those who consumed lower conservative media were higher on vaccine intention and individual vs. collective frames did not have a strong impact on these people. However, within those participants who scored high on conservative media use, the individual frame had a higher impact on COVID-19 vaccine intention.

When the message highlighted the impact of the vaccine on themselves, these individuals were more impacted by that message. Perhaps these findings can be explained by research that shows that in general, conservative thinking have less empathy for a larger community and are more involved about themselves and a closer circle of family members and friends (Brueck, 2018; Crawford et al., 2013). As a result, the individual frame may speak closely to conservatives since this message appeals to the benefits to the individual and not the community. Of course, there can be other factors that describe conservative vs. liberal thinking. The findings show that vaccine promotion messages will have to pay attention to these nuances and target different audiences with specific messages. For example, public health organizations should keep in mind that people with different ideologies and worldviews may resonate with different messages about the vaccine. It will help to have specific types of content to reach out to these individuals.

The findings with attitudes as a moderator revealed similar nuances in the impact of message framing. The results show that those individuals who held positive attitudes toward vaccination were not affected by individual vs. collective frames. These people were high on vaccine intention irrespective of the type of message they read. However, those individuals who held negative attitudes toward vaccination showed higher intention to vaccinate in the individual framed message condition. The findings indicate that once again the individual frame is more persuasive for these participants. Overall, these findings make sense. Those people who used more conservative media and held negative attitudes toward vaccines are perhaps alike in nature and held similar values. In both models, individual frame was more effective, which shows that these individuals resonated more with the messages that highlight the importance of taking care of their own health, and not the communities' health. These findings could perhaps be linked to psychological dispositions as well (e.g., Luyten et al., 2014). Prior research has also shown that vaccine sceptics scored lower on collectivism and showed significantly lower "disposition to see others as equals" (Luyten et al., 2014, p. 310) compared to non-sceptics.

These findings are critical for vaccine promotion research. Often it might be considered that people do not have enough information about a particular vaccine, which is why they are hesitant about vaccines. That may be true in some cases, but the findings from the current study also show that it might not necessarily always be about knowledge of the vaccines. People who are hesitant about vaccines may be because of their world views and political ideologies. Vaccine promotion messages should pay attention to these individual characteristics. Besides informing people and increasing knowledge, health organizations should also focus on how the information is shared. The findings from the current study as well as past research (e.g., Nan, 2012a; Park, 2012; Xiao \& Borah, 2021), show that the type of message can play a significant role.

As with all research, this study comes with some caveats. The source of the Facebook post was CDC. Future research can test these messages using another source, to disregard any biases about CDC. The experiment tests the effects of one message, examining multiple messages will be helpful for future research. Although this study uses 
a single message, the experiment included four different message frames. It is important to note that those who score higher on conservative media use, might also be more conservative ideologically. The current study controlled for party ID, but future research can delve deeper into these relationships. The current study tests vaccine intention. Future research should also examine COVID19 vaccination behavior. The vaccine intention measure consisted of three items used in prior research such as Nan (2012a). Future research may include additional items to vaccine intention measures. The attitude toward vaccination measure consisted of a single item and was also adapted from past research (e.g., Nan, 2012a). Future research should include additional items to strengthen this measure. The media use variables included three major outlets, but there are many examples of partisan media in the current landscape. Future research can incorporate more outlets.

Despite some of these limitations, the current study takes an attempt to examine the relationship between message frames, partisan media, and attitudes on COVID-19 vaccination. These findings have crucial implications for public health. At a time when the COVID-19 pandemic has become a highly politicized issue, understanding how to reach different groups of individuals to promote the vaccine is critical. Public health organizations should use messages that can help to reach a specific group of people. These findings can help in promoting the COVID-19 vaccine by reaching people on different political spectrum.

Supplementary Information The online version contains supplementary material available at https://doi.org/10.1007/s12144-022-02851-3.

\section{Authors' Contributions Not applicable.}

Funding The research was not supported by any funding.

Data Availability Data can be made available upon request.

Code Availability Not applicable.

\section{Declarations}

The study was approved as exempt by the Institutional Research Board of Washington State University in the U.S.

Ethics Approval The study was approved as exempt by the Institutional Research Board of a large Washington State University in the U.S.

Consent to Participate Each participant read and accepted the consent form before participation.

Consent for Publication I approve publication of this manuscript if accepted.

Conflicts of Interest/Competing Interests Not applicable.

\section{References}

Bauder, D. (2021). Conservative media offers mixed messages on COVID-19 vaccine. Retrieved from https://apnews.com/article/ joe-biden-health-government-and-politics-arts-and-entertainm ent-media-74f36fead348661aeba170422381a972. Accessed 23 Jan 2022

Baum, M. A. (2011). Red state, blue state, flu state: Media self-selection and partisan gaps in swine flu vaccinations. Journal of Health Politics, Policy and Law, 36(6), 1021-1059.

Berinsky, A. J., Huber, G. A., \& Lenz, G. S. (2012). Evaluating online labor markets for experimental research: Amazon.com's Mechanical Turk. Political Analysis, 20, 351-368. https://doi.org/10.1093/ pan/mpr057.

Bigman, C. A., Cappella, J. N., \& Hornik, R. C. (2010). Effective or ineffective: Attribute framing and the human papillomavirus (HPV) vaccine. Patient Education and Counseling, 81(Suppl.), S70-S76. https://doi.org/10.1016/j.pec.2010.08.014

Blume, S. (2006). Anti-vaccination movements and their interpretations. Social Science \& Medicine, 62(3), 628-642. https://doi.org/ 10.1016/j.socscimed.2005.06.020

Böhm, R., Betsch, C., Korn, L., \& Holtmann, C. (2016). Exploring and promoting prosocial vaccination: A cross-cultural experiment on vaccination of health care personnel. BioMed Research International. https://doi.org/10.1155/2016/6870984

Borah, P. (2011). Conceptual issues in framing theory: A systematic examination of a decade's literature. Journal of Communication, 61(2), 246-263. https://doi.org/10.1111/j.1460-2466.2011. 01539.x

Briley, D. A., Rudd, M., \& Aaker, J. (2017). Cultivating optimism: How to frame your future during a health challenge. Journal of Consumer Research, 44(4), 895-915. https://doi.org/10.1093/jcr/ucx075

Brueck, H. (2018). Scientists have discovered two simple psychological differences that make you liberal or conservative. Retrieved from https://www.businessinsider.com/liberals-and-conservatives-proce ss-disgust-and-empathy-differently-2018-1. Accessed 23 Jan 2022

Buhrmester, M., Kwang, T., \& Gosling, S. D. (2016). Amazon's mechanical Turk: A new source of inexpensive, yet high-quality data? In A. E. Kazdin (Ed.), Methodological issues and strategies in clinical research. American Psychological Association (p. 133-139). https://doi.org/10.1037/14805-009

Centers for Disease Control and Prevention. (2018). Vaccines: VacGen/Imz BASICS main page. Retrieved from https://www.cdc. gov/vaccines/vac-gen/imz-basics.htm

Chanel, O., Luchini, S., Massoni, S., \& Vergnaud, J. C. (2011). Impact of information on intentions to vaccinate in a potential epidemic: Swine-origin Influenza A (H1N1). Social Science \& Medicine, 72(2), 142-148. https://doi.org/10.1016/j.socscimed.2010.11.018

Chen, T., Dai, M., Xia, S., \& Zhou, Y. (2021). Do messages matter? Investigating the combined effects of framing, outcome uncertainty, and number format on COVID-19 vaccination attitudes and intention. Health Communication, 1-8. https://doi.org/10.1080/ 10410236.2021.1876814

Crawford, J., Modri, S., \& Motyl, M. (2013). Bleeding-heart liberals and hard-hearted conservatives: Subtle political dehumanization through differential attributions of human nature and human uniqueness traits. Available at SSRN 2310758.

D’Angelo, P. (2002). News framing as a multiparadigmatic research program: A response to Entman. Journal of Communication, 52(4), 870-888. https://doi.org/10.1111/j.1460-2466.2002.tb025 78. $\mathrm{x}$

Dillard, J. P. (2011). An application of the integrative model to women's intention to be vaccinated against HPV: Implications for message design. Health Communication, 26(5), 479-486. https://doi. org/10.1080/10410236.2011.554170 
Dong, E., Du, H., \& Gardner, L. (2020). An interactive web-based dashboard to track COVID-19 in real time. The Lancet Infectious Diseases, 20(5), 533-534. https://doi.org/10.1016/S14733099(20)30120-1

Dutta, M. J. (2007). Communicating about culture and health: Theorizing culture-centered and cultural sensitivity approaches. Communication Theory, 17(3), 304-328. https://doi.org/10.1111/j. 1468-2885.2007.00297.x

Earley, P. C. (1989). Social loafing and collectivism: A comparison of the United States and the People's Republic of China. Administrative Science Quarterly, 565-581. https://doi.org/10.2307/2393567

Elkind, E. (2020). Americans' "vaccine hesitancy" could be a barrier to defeating COVID-19, doctor warns. Retrieved from https://www. cbsnews.com/news/covid-19-american-vaccine-hesitancy-doctor/

Erdfelder, E., Faul, F., \& Buchner, A. (1996). GPOWER: A general power analysis program. Behavior Research Methods, Instruments, \& Computers, 28(1), 1-11.

Finkelstein, M. A. (2010). Individualism/collectivism: Implications for the volunteer process. Social Behavior and Personality: An International Journal, 38(4), 445-452. https://doi.org/10.2224/ sbp.2010.38.4.445

Franck, T. (2020). Trump says the coronavirus is the Democrats' 'new hoax'. https://www.cnbc.com/2020/02/28/trump-says-the-coron avirus-is-the-democrats-new-hoax.html

Frisby, C. M. (2018). "Oh, See What We Say:” A Content Analysis of Partisan Media's Framing of the "take a knee" silent protest by the NFL. American International Journal of Humanities and Social Science, 4, 6-18.

Gainforth, H. L., Cao, W., \& Latimer-Cheung, A. E. (2012). Message framing and parents intentions to have their children vaccinated against HPV. Public Health Nursing, 29(6), 542-552. https://doi. org/10.1111/j.1525-1446.2012.01038.x

Gerend, M. A., \& Shepherd, J. E. (2012). Predicting human papillomavirus vaccine uptake in young adult women: Comparing the health belief model and theory of planned behavior. Annals of Behavioral Medicine, 44(2), 293-293. https://doi.org/10.1007/ s12160-012-9366-5

Gollust, S. E., Attanasio, L., Dempsey, A., Benson, A. M., \& Fowler, E. F. (2013). Political and news media factors shaping public awareness of the HPV vaccine. Women's Health Issues, 23(3), e143-e151. https://doi.org/10.1016/j.whi.2013.02.001

Gollust, S. E., Dempsey, A., Lantz, P. M., Ubel, P. A., \& Fowler, E. F. (2010). Controversy undermines support for state mandates on the human papillomavirus vaccine. Health Affairs, 29, 2041-2046. https://doi.org/10.1377/hlthaff.2010.0174

Gollust, S. E., LoRusso, S. M., Nagler, R. H., \& Fowler, E. F. (2016). Understanding the role of the news media in HPV vaccine uptake in the United States: Synthesis and commentary. Human Vaccines \& Immunotherapeutics, 12(6), 1430-1434. https://doi.org/ 10.1080/21645515.2015.1109169

Guidry, J. P., Laestadius, L. I., Vraga, E. K., Miller, C. A., Perrin, P. B., Burton, C. W., ... \& Carlyle, K. E. (2020). Willingness to get the COVID-19 Vaccine with and without emergency use authorization. American Journal of Infection Control.

Hayes, A. F. (2013). Introduction to mediation, moderation, and conditional process analysis. New York, NY: Guilford Press.

Jamison, A. M., Broniatowski, D. A., Dredze, M., Sangraula, A., Smith, M. C., \& Quinn, S. C. (2020). Not just conspiracy theories: Vaccine opponents and pro-ponents add to the COVID-19 'infodemic'on Twitter. Harvard Kennedy School Misinformation Review, 1(3)

Kahneman, D., \& Tversky, A. (1984). Choices, values, and frames. American Psychologist, 39(4), 341-350. https://doi.org/10.1037/ 0003-066X.39.4.341

Law, T. (2020). There Isn't a COVID-19 Vaccine Yet. But Some Are Already Skeptical About It. Retrieved from https://time.com/ 5836800/covid-19-vaccine-skepticism/?utm_source=twitter\& utm_medium $=$ social\&utm_campaign=editorial\&utm_term $=$ health_covid-19\&linkId $=88903767$

Leader, A. E., Weiner, J. L., Kelly, B. J., Hornik, R. C., \& Cappella, J. N. (2009). Effects of information framing on human papillomavirus vaccination. Journal of Women's Health, 18(2), 225-233. https://doi.org/10.1089/jwh.2007.0711

Lehmann, B. A., Ruiter, R. A., Wicker, S., Chapman, G., \& Kok, G. (2015). Medical students' attitude towards influenza vaccination. BMC Infectious Diseases, 15(1), 185.

Levendusky, M. S. (2013). Why do partisan media polarize viewers? American Journal of Political Science, 57(3), 611-623.

Luyten, J., Desmet, P., Dorgali, V., Hens, N., \& Beutels, P. (2014). Kicking against the pricks: Vaccine sceptics have a different social orientation. The European Journal of Public Health, 24(2), 310-314

McRee, A. L., Reiter, P. L., Chantala, K., \& Brewer, N. T. (2010). Does framing human papillomavirus vaccine as preventing cancer in men increase vaccine acceptability? Cancer Epidemiology, Biomarkers and Prevention, 19, 1937-1944. https://doi.org/10.1158/ 1055-9965.EPI-09-1287

Motta, M., Stecula, D., \& Farhart, C. (2020). How right-leaning media coverage of COVID-19 facilitated the spread of misinformation in the early stages of the pandemic in the US. Canadian Journal of Political Science, 1-8. https://doi.org/10.1017/S0008423920000396

Nan, X., Xie, B., \& Madden, K. (2012). Acceptability of the H1N1 vaccine among older adults: The interplay of message framing and perceived vaccine safety and efficacy. Health Communication, 27(6), 559-568. https://doi.org/10.1080/10410236.2011.617243

Nan, X. (2012a). Relative persuasiveness of gain-versus loss-framed human papillomavirus vaccination messages for the present-and future-minded. Human Communication Research, 38(1), 72-94. https://doi.org/10.1111/j.1468-2958.2011.01419.x

Nan, X. (2012b). Communicating to young adults about HPV vaccination: Consideration of message framing, motivation, and gender. Health Communication, 27(1), 10-18. https://doi.org/10.1080/ 10410236.2011.567447

Nyhan, B., Reifler, J., Richey, S., \& Freed, G. L. (2014). Effective messages in vaccine promotion: A randomized trial. Pediatrics, 133(4), e835-e842. https://doi.org/10.1542/peds.2013-2365

O’Keefe, D. J., \& Jensen, J. D. (2008). Do loss-framed persuasive messages engender greater message processing than do gain-framed messages? A meta-analytic review. Communication Studies, 59(1), 51-67. https://doi.org/10.1080/10510970701849388

O'Keefe, D. J., \& Wu, D. (2012). Gain-framed messages do not motivate sun protection: A meta-analytic review of randomized trials comparing gain-framed and loss-framed appeals for promoting skin cancer prevention. International Journal of Environmental Research and Public Health, 9(6), 2121-2133. https://doi.org/10. 3390/ijerph9062121

Oyserman, D., Coon, H. M., \& Kemmelmeier, M. (2002). Rethinking individualism and collectivism: Evaluation of theoretical assumptions and meta-analyses. Psychological Bulletin, 128(1), 3-72. https://doi.org/10.1037/0033-2909.128.1.3

Oyserman, D., \& Lee, S. W. S. (2008). Does culture influence what and how we think? Effects of priming individualism and collectivism. Psychological Bulletin, 134(2), 311-342. https://doi.org/10.1037/ 0033-2909.134.2.311

Park, S. (2012). The effects of message framing and risk perceptions for HPV vaccine campaigns: Focus on the role of regulatory fit. Health Marketing Quarterly, 29(4), 283-302. https://doi.org/10. 1080/07359683.2012.732847

Pittman, M. (2020). Accountability moderates the effects of egoistic and altruistic appeals in prosocial messages. Journal of Consumer Marketing, 37(7), 807-820. https://doi.org/10.1108/ JCM-07-2018-2751 
Rabb, N., Glick, D., Houston, A., Bowers, J., \& Yokum, D. (2021). No evidence that collective-good appeals best promote COVIDrelated health behaviors. Proceedings of the National Academy of Sciences, 118(14).

Ratanasiripong, N. T., Cheng, A. L., \& Enriquez, M. (2013). What college women know, think, and do about human papillomavirus (HPV) and HPV vaccine. Vaccine, 31(10), 1370-1376. https://doi. org/10.1016/j.vaccine.2013.01.001

Rupar, A. (2020). Hannity claims he's "never called the virus a hoax" 9 days after decrying Democrats' "new hoax." Retrieved from. https://www.vox.com/2020/3/20/21186727/hannity-coronaviruscoverage-fox-news

Scheufele, D. A. (1999). Framing as a theory of media effects. Journal of Communication, 49(1), 103-122.

Slisco, A. (2020). Right-wing news site warns readers not to get hypothetical coronavirus vaccine because vaccines are a 'scam' Retrieved from https://www.newsweek.com/right-wing-news-sitewarns-readers-not-get-hypothetical-coronavirus-vaccine-becausevaccines-are-1491579

Thompson, M. G., Pierse, N., Huang, Q. S., Prasad, N., Duque, J., Newbern, E. C., \& Mcarthur, C. (2018). Influenza vaccine effectiveness in preventing influenza-associated intensive CARE admissions and attenuating severe disease among adults in new ZEALAND 2012-2015. Vaccine, 36(39), 5916-5925. https://doi. org/10.1016/j.vaccine.2018.07.028

Triandis, H. C. (2001). Individualism-collectivism and personality. Journal of Personality, 69(6), 907-924.

Tversky, A., \& Kahneman, D. (1981). The framing of decisions and the psychology of choice. Science, 211(4481), 453-458. https:// doi.org/10.1126/science. 7455683

The United Nations Children's Fund, (2019). Immunization. https:// data.unicef.org/topic/child-health/immunization/.

Uskul, A. K., Sherman, D. K., \& Fitzgibbon, J. (2009). The cultural congruency effect: Culture, regulatory focus, and the effectiveness of gain-vs. loss-framed health messages. Journal of Experimental Social Psychology, 45(3), 535-541. https://doi.org/10.1016/j.jesp. 2008.12.005
Von Sikorski, C., \& Matthes, J. (2019). Framing effects in health communication. In C. Rossmann \& M. Hastall (Eds.), Handbook of Health Communication. (pp. 1-13). Wiesbaden, Germany: Springer VS.

West, D. M. (2001). The Rise and Fall of the Media Establishment. St. Martin's Press.

Wheldon, C. W., Daley, E. M., Walsh-Buhi, E. R., Baldwin, J. A., Nyitray, A. G., \& Giuliano, A. R. (2018). An integrative theoretical framework for HPV vaccine promotion among male sexual minorities. American Journal of Men's Health, 12(5), 1409-1420. https://doi.org/10.1177/1557988316652937

Workman, J. E., \& Lee, S. H. (2011). Materialism, fashion consumers and gender: A cross-cultural study. International Journal of Consumer Studies, 35(1), 50-57. https://doi.org/10.1111/j.14706431.2010.00935.x

World Health Organization. (n.d). Six common misconceptions about immunization. Retrieved from https://www.who.int/vaccine safety/initiative/detection/immunization_misconceptions/e/ index $2 . \mathrm{html}$.

Xiao, X., \& Wong, R. (2020). Vaccine hesitancy and perceived behavioral control: A meta-analysis. Vaccine. https://doi.org/10.1016/j. vaccine.2020.04.076

Xiao, X., \& Borah, P. (2021). Do norms matter? Examining normbased messages in HPV vaccination promotion. Health Communication, 36(12), 1476-1484. https://doi.org/10.1080/10410236. 2020.1770506

Yanovitzky, I. (2002). Effects of news coverage on policy attention and actions: A closer look into the media-policy connection. Communication Research, 29(4), 422-451. https://doi.org/10.1177/00936 50202029004003

Publisher's Note Springer Nature remains neutral with regard to jurisdictional claims in published maps and institutional affiliations. 